\title{
Estimación de parámetros de los equilibrios líquido-líquido ternarios entre 2-etil-1-hexanol y agua con butanol, ácido acético y etanol usando UNIQUAC
}

\author{
Víctor H. Grisales-Díaz ${ }^{1,3 *}$, Oscar A. Prado-Rubio ${ }^{1}$ y Gerard Olivar-Tost ${ }^{2,4}$ \\ (1) Fac. De Ingeniería y arquitectura, Dpto. de Ingeniería Química, Universidad Nacional de Colombia - Sede Manizales, \\ Cra. 27 No.64-60, Manizales, Colombia. (correo-e: vhgrisalesd@unal.edu.co, oaprador@unal.edu.co) \\ (2) Fac. De Ciencias Exactas y Naturales, Dpto. de Matemática y Estadística, Universidad Nacional de Colombia - Sede \\ Manizales, Cra. 27 No.64-60, Manizales, Colombia. (correo-e: golivart@unal.edu.co) \\ (3) Fac. Ciencias de la Salud. Dpto. Microbiología. Universidad Libre, Grupo de investigación en Microbiología y \\ Biotecnología MICROBIOTEC, Belmonte Avenida Las Américas, Pereira, Colombia. \\ (correo-e: victorh.grisalesd@unilibre.edu.co) \\ (4) Departamento de Ciencias Naturales y Tecnología, Universidad de Aysén, Coyhaique, Chile. \\ (correo-e: gerard.olivar@uaysen.cl)
}

* Autor a quien debe ser dirigida la correspondencia

Recibido Ene. 2, 2020; Aceptado Mar. 3, 2020; Versión final May. 6, 2020, Publicado Oct. 2020

\begin{abstract}
Resumen
El objetivo de esta investigación es simular con mayor precisión los equilibrios que se generan en la separación de butanol usando el extractante 2-etil-1-hexanol $(2 \mathrm{E} 1 \mathrm{H})$. Los parámetros binarios fueron ajustados usando el método de algoritmo genético y un optimizador para ajuste no-lineal, 'Isqcurvefit', de MATLAB ${ }^{\circledR}$. Los productos considerados en los equilibrios ternarios fueron butanol, acetona, etanol, y ácido acético y fueron simulados usando UNIQUAC. En el ajuste de los equilibrios se alcanzó una desviación media de la raíz cuadrada (RMSD) de 1.4. El cual es 4.7 veces menor que el RMSD (\%) obtenido usando UNIQUAC y los parámetros reportados en el principal software para la simulación de procesos químicos industriales, Aspen Plus $\mathrm{V} 10^{\circledR}$. Se recomienda usar la mayor cantidad disponible de datos experimentales de equilibrios líquido-líquido para poder alcanzar simulaciones con el más bajo error posible. No se recomienda el uso de los parámetros disponibles de Aspen Plus $\mathrm{V}_{10}{ }^{\circledR}$ para la extracción de butanol mediante $2 \mathrm{E} 1 \mathrm{H}$.
\end{abstract}

\section{Estimation of parameters for ternary liquid-liquid equilibria of 2-ethyl-1-hexanol and water with butanol, acetic acid, and ethanol using UNIQUAC}

\begin{abstract}
The main objective of this research study is to model the equilibria in the separation of butanol using the extractant 2-ethyl-1-hexanol $(2 \mathrm{E} 1 \mathrm{H})$. Binary parameters were fitted using MATLAB's default genetic algorithm function and a solver for nonlinear curve-fitting ('Isqcurvefit'). The products assessed in the ternary equilibria were ethanol, butanol, acetone, and acetic acid and were simulated using UNIQUAC. The root mean square deviation (RMSD) of the prediction for these ternary liquid-liquid equilibria was 1.4, which is 4.7 times lower than that achieved by using Aspen Plus $V 10^{\circledR}$ parameters, a top commercial software for simulating industrial chemical processes. It is recommended to use the maximum possible amount of experimental data of liquid-liquid equilibria to achieve simulations with the lowest error possible. It is not recommended to use Aspen Plus $\mathrm{V}_{1} 0^{\circledR}$ parameters for butanol extractions using $2 \mathrm{E} 1 \mathrm{H}$.
\end{abstract}




\section{INTRODUCCIÓN}

El butanol es un posible biocombustible y un químico de alta demanda mundial (Karimi-Alavijeh y Karimi, 2019). A nivel industrial, la producción biológica de butanol es fabricada usando la fermentación $A B E$ (acetona, butanol y etanol). La fermentación ABE es realizada por diferentes microorganismos, tales como C. acetobutylicum, y tiene una alta demanda energética en la separación y baja productividad por la alta inhibición del producto (Grisales-Díaz y Olivar-Tost, 2017). Una de las alternativas para mejorar el desempeño de esta fermentación es a través de la intensificación de procesos usando recuperación in situ de producto (ISPR), (Boock et al., 2019; Naidoo et al., 2018). Las principales ventajas de este tipo de reactores son sus bajos requerimientos de agua y energéticos, y su elevada productividad (Grisales-Díaz y Olivar-Tost, 2019).

Una de las ventajas relevantes en los sistemas con extracción in situ, es la capacidad de incrementar la productividad o de disminuir el tamaño del reactor al disminuir la inhibición por producto. No obstante, las unidades de recuperación necesarias en este sistema representan tanto costos como requerimientos energéticos adicionales en relación con el proceso convencional (Singh et al., 2019). Por ende, es fundamental determinar las condiciones óptimas de operación que permitan minimizar el tamaño del reactor, maximizar el rendimiento de la fermentación, así como minimizar los requerimientos totales de energía (Singh et al., 2019). Debido al alto número de variables involucradas, son fundamentales estrategias de simulación que permitan modelar el comportamiento de procesos industriales con una alta precisión (Bedade et al., 2019; Forero y Velásquez, 2019). Entre las técnicas de recuperación in situ, los requerimientos energéticos de las unidades que usan extractantes, extracción y pertracción, han sido reportados por Qureshi et al., 2005, Outram et al., 2016 y Groot et al., 1992 con uno de los requerimientos energéticos más bajos entre las distintas tecnologías evaluadas para la recuperación de butanol.

Un listado de los extractantes que pueden ser usados en este proceso, es mostrado en la Tabla 1. En la extracción de butanol, el solvente o extractante más estudiado en la literatura es el alcohol oleico ( $\mathrm{Lu}$ y Li, 2014). Este solvente ha sido ampliamente investigado porque no es tóxico para el microorganismo, tiene aceptables coeficientes de distribución (i.e. entre 3 y 4), y altas selectividades (Matsumura et al., 1988), ver Tabla 1. Sin embargo, ha sido sugerido recientemente por (Dalle-Ave y Adams, 2018) que el 2-etil-1-hexanol $(2 \mathrm{E} 1 \mathrm{H})$ es la mejor opción por su alta selectividad o bajo coeficiente de reparto de agua y alto coeficiente de distribución de butanol, ver Tabla 1.

Tabla 1: Coeficientes de partición de butanol y agua usando diferentes solventes orgánicos a $25^{\circ} \mathrm{C}$ y 1 atm

\begin{tabular}{|l|l|l|l|}
\hline Solvente & $\begin{array}{l}\text { Coeficiente de reparto } \\
\text { del butanol }\end{array}$ & $\begin{array}{l}\text { Coeficiente de } \\
\text { reparto del agua }\end{array}$ & Referencias \\
\hline Octano & 0.30 & $7.2 \mathrm{E}-05$ & (Groot et al., 1990; Leo et al., 1971) \\
\hline Aceite de sésamo & 0.30 & $1.3 \mathrm{E}-03$ & (Groot et al., 1990) \\
\hline Laurato de metilo & 1.82 & 0.26 & (Groot et al., 1990) \\
\hline Maleato de dibutilo & 2.00 & 0.66 & (Groot et al., 1990) \\
\hline Aceite de castor & 2.57 & $9.6 \mathrm{E}-03$ & (Groot et al., 1990) \\
\hline Alcohol oleico & 3.02 & 0.02 & (Davison y Thompson, 1993) \\
\hline Acetato de hexilo & 3.63 & 0.72 & (Groot et al., 1990) \\
\hline Dodecanol & 6.03 & 0.04 & (Groot et al., 1990) \\
\hline Octanol & 7.59 & 0.078 & (Groot et al., 1990; Leo et al., 1971) \\
\hline Hexanol & 12.0 & 0.076 & (Groot et al., 1990) \\
\hline 2-etil-1-hexanol $\left(36^{\circ} \mathrm{C}\right)$ & 7.95 & 0.025 & (González-Peñas et al., 2014) \\
\hline
\end{tabular}

La extracción de butanol usando solventes involucra una o más etapas de equilibrio líquido-líquido. El equilibrio es alcanzado cuando las actividades de todas las fases del sistema son iguales. Consecuentemente, la simulación de ISPR por extracción necesita de modelos que simulen la actividad, y permitan, así, determinar las composiciones de equilibrio. Entre estos modelos, UNIQUAC es uno de los más usados. Aunque el modelo de UNIQUAC puede ser usado para modelar equilibrios complejos, este modelo tiene como requerimiento que los parámetros de interacción binarios presentes en el modelo sean determinados a partir de la información experimental disponible (Rezaei et al., 2018). Los datos experimentales usados típicamente para determinar estos parámetros son equilibrios líquido-líquido de dos o tres componentes.

En lo referente a la extracción de butanol con $2 \mathrm{E} 1 \mathrm{H}$, ya que el butanol es extraído de una solución acuosa, son requeridos de equilibrios ternarios de $2 \mathrm{E} 1 \mathrm{H}$, agua y butanol. Estos equilibrios ternarios han sido previamente reportados por Ghanadzadeh y Ghanadzadeh, 2004. Otra importante consideración en la 
simulación de la extracción de butanol es la presencia de los coproductos de la fermentación, tales como el ácido acético, la acetona y el etanol. Por lo tanto, también han sido reportados datos de experimentos de equilibrios ternarios que involucren $2 \mathrm{E} 1 \mathrm{H}$ y agua con estos coproductos ( $\mathrm{H}$ Ghanadzadeh et al., 2004; $\mathrm{H}$. Ghanadzadeh et al., 2004; Ghanadzadeh y Ghanadzadeh, 2003).

En estos trabajos previos, los parámetros de interacción binaria fueron estimados y fue corroborado el poder predictivo del modelo de UNIQUAC para sus respectivos equilibrios. No obstante, la estimación de los parámetros de interacción binaria del modelo UNIQUAC fue llevada a cabo separadamente usando de manera individual cada uno de los equilibrios ternarios. Esto es importante de recalcar porque existe parámetros en común para cada uno de los equilibrios ternarios, los cuales están relacionados a los parámetros de interacción binaria $\left(\tau_{i j}\right)$ entre $2 \mathrm{E} 1 \mathrm{H}$ y agua. Dado que han sido obtenidos a partir de datos experimentales de equilibrios ternarios con diferentes mezclas de componentes, esto ha conllevado a inconsistencias en la estimación de su magnitud.

En este sentido, las inconsistencias de la magnitud de los parámetros son observadas en la Fig. 1. En la Fig. 1 se observa que el efecto de los parámetros de interacción binaria $\left(T_{i j}\right)$ entre $2 \mathrm{E} 1 \mathrm{H}$ y agua con la temperatura puede llegar incluso a tener una diferente pendiente. En la Fig. 1 los parámetros reportados de interacción binaria $(\mathrm{Tij})$ usando diferentes equilibrios ternarios. Los parámetros para el equilibrio acetona, $2 \mathrm{E} 1 \mathrm{H}$ y agua fueron reportados por Ghanadzadeh et al., 2004a y los parámetros para el equilibrio etanol, $2 \mathrm{E} 1 \mathrm{H}$ y agua fueron reportados por Ghanadzadeh y Ghanadzadeh, 2003. Mientras, Los parámetros para el equilibrio butanol, 2-etil-1hexanol $(2 \mathrm{E} 1 \mathrm{H})$ y agua fueron reportados por Ghanadzadeh y Ghanadzadeh, 2004b.
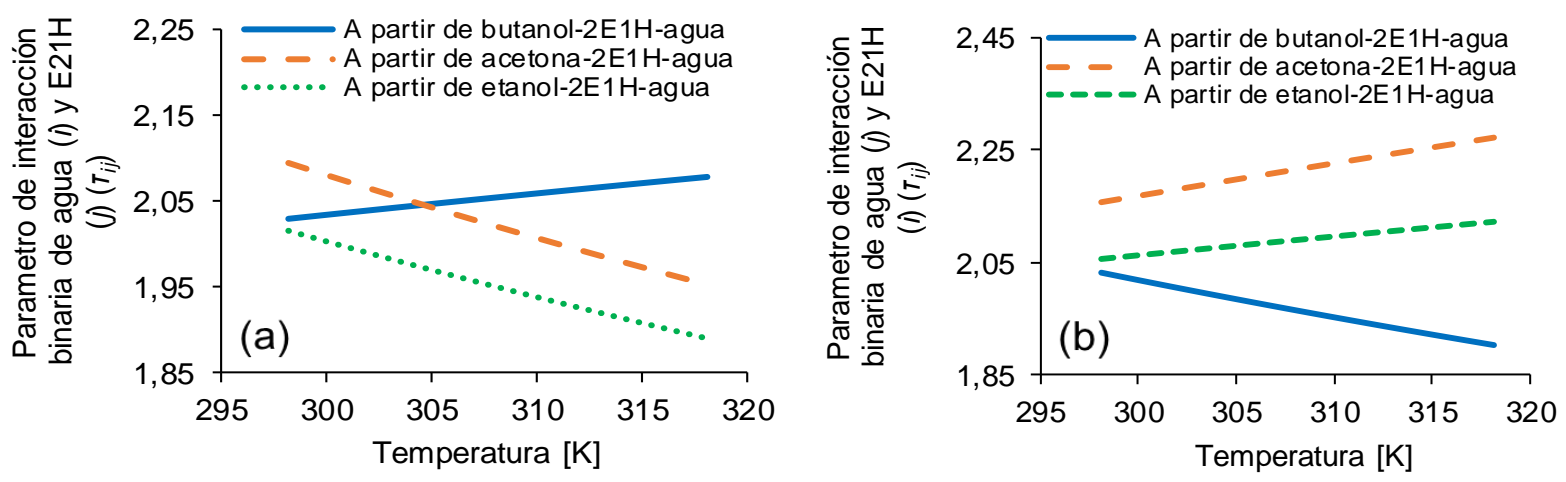

Fig. 1: Efecto de los parámetros de interacción binaria $\left(\tau_{i j}\right)$ entre $2 \mathrm{E} 1 \mathrm{H}$ y agua con la temperatura.

Por las discrepancias observadas en los parámetros binarios $\left(\tau_{i j}\right)$ entre $2 \mathrm{E} 1 \mathrm{H}$ y agua, en este trabajo se realiza una estimación de los parámetros usando de manera simultánea cada uno de los equilibrios ternarios de $2 \mathrm{E} 1 \mathrm{H}$ y agua, con los productos de la producción de butanol. Los productos considerados fueron butanol, acetona, etanol y ácido acético. Lo cual es contrario a lo realizado en la literatura para estos datos de equilibrio, dado que los parámetros de interacción binaria que han sido reportados en trabajos anteriores fueron encontrados usando de manera individual los respectivos equilibrios ternarios con $2 \mathrm{E} 1 \mathrm{H}, \mathrm{y}$ no fueron usados simultáneamente todos, tal como es propuesto en este trabajo. En consecuencia, los parámetros determinados del modelo de actividad de UNIQUAC en este trabajo permiten estimar los equilibrios multicomponente con mayor exactitud, y serán de utilidad para optimizar la producción de butanol con la extracción in situ, usando el solvente $2 \mathrm{E} 1 \mathrm{H}$.

\section{EL MODELO DE EQUILIBRIO}

La composición de cada uno de los componentes de las dos fases del equilibrio líquido-líquido es alcanzado cuando las actividades de las dos fases son iguales. Teniendo en cuenta que la sumatoria de las composiciones debe ser igual a 1, el equilibrio de fases es estimado mediante las siguientes ecuaciones:

$$
\begin{aligned}
& \left(x_{i} \cdot \gamma_{i}\right)_{I}=\left(x_{i} \cdot \gamma_{i}\right)_{I I} \\
& \left(\sum x_{i}\right)_{I}=\left(\sum x_{i}\right)_{I I}=1
\end{aligned}
$$

donde, la composición molar de cada componente es descrita usando la variable $x_{i}$. Los términos en el lado izquierdo en la Eq. (1) son la actividad de la fase $I$ y los del lado derecho de la Eq. (2) representan la actividad de la fase II. Los coeficientes de actividad $\left(\gamma_{i}\right)$ de la fase $I$ y II son determinados usando el modelo de UNIQUAC (Abrams y Prausnitz, 1975), tal como es descrito a continuación: 
$\ln \left(\gamma_{i}\right)=\ln \left(\gamma_{i}\right)^{C}+\ln \left(\gamma_{i}\right)^{R}$

La parte combinacional $\left(\ln \left(\gamma_{i}\right) \mathcal{C}\right)$ y la residual $\left(\ln \left(\gamma_{i}\right)^{R}\right)$ del modelo UNIQUAC son debidas a la forma y energía de las moléculas, respectivamente, y son descritas a continuación:

$\ln \left(\gamma_{i}\right)^{C}=\ln \left(\frac{\Phi_{i}}{x_{i}}\right)+\frac{z}{2} q_{i}\left(\frac{\theta_{i}}{\Phi_{i}}\right)+l_{i}-\frac{\Phi_{i}}{x_{i}} \sum_{j=1}^{c} x_{j} l_{j}$
$\ln \left(\gamma_{i}\right)^{R}=q_{i}\left[1-\ln \left(\sum_{j=1}^{c} \theta_{j} \tau_{j i}\right)-\sum_{j=1}^{c}\left(\frac{\theta_{j} \tau_{j i}}{\sum_{k=1}^{c} \theta_{k} \tau_{k j}}\right)\right]$

donde $z$ es igual a 10. Los parámetros $l_{i}$, y los parámetros $\Phi_{i}$ (fracción de segmento) y $\theta$ (fracción de área) son calculados a partir de los parámetros estructurales, $r_{i}$ y $q_{i}$, usando cada una de las siguientes ecuaciones:

$l_{i}=\frac{Z}{2}\left(r_{i}-q_{i}\right)-\left(r_{i}-1\right)$

$\Phi_{i}=\frac{x_{i} r_{i}}{\sum_{i=1}^{c} x_{i} r_{i}} ; \theta_{i}=\frac{x_{i} q_{i}}{\sum_{i=1}^{c} x_{i} q_{i}}$

Los parámetros para cada componente de fracción de volumen ri y de área de superficie molecular $q_{i}$ están reportados en la Tabla 2 y no son ajustables, donde $c$ es el número de componentes. En este trabajo, $c$ es igual a 3, dado que se están estudiando equilibrios ternarios.

Tabla 2: Parámetros estructurales de UNIQUAC extraídos de Aspen Plus V10®

\begin{tabular}{|c|c|c|c|c|c|c|}
\hline Parámetro & 1-Butanol & Acetona & Etanol & Ácido acético & Agua & 2-ethyl-1-hexanol \\
\hline$r_{i}$ & 3.45 & 2.54 & 2.11 & 2.10 & 0.92 & 6.15 \\
\hline$q_{i}$ & 3.05 & 2.34 & 1.97 & 2.07 & 1.40 & 5.02 \\
\hline
\end{tabular}

Los parámetros de interacción binaria son ajustables y se calculan a partir de la siguiente ecuación:

$\tau_{j i}=\exp \left(a_{i j}+{ }^{b_{i j}} /_{T}+c_{i j} \cdot \log (T)\right)$

donde, los parámetros $a_{i j}$ y $b_{i j}$, son dependientes de la temperatura, $\mathrm{T}(\mathrm{K})$, y fueron ajustados. El parámetro cij fue asumido cero para todos los parámetros de interacción binaria, excepto para la combinación de agua y butanol, ya que los parámetros que describen mejor el equilibrio líquido-líquido binario incluyen ese parámetro (Grisales-Díaz y Olivar-Tost, 2018). Para la minimización del error en la estimación de los parámetros, después de asumidos los parámetros de interacción binarios, se estiman las composiciones de equilibrio para cada una de los experimentos (Sørensen et al., 1979). De esta manera, se asegura que los parámetros obtenidos predigan cada una de las composiciones en el equilibrio y cumplan con el balance de materia. La función objetivo fue la desviación cuadrática de la media ( $R M S D$, de sus siglas en inglés) entre cada una de las composiciones experimentales y estimadas mediante el modelo de UNIQUAC. El RMSD fue estimado mediante la siguiente ecuación:

$\operatorname{RMSD}(\%)=100 \cdot\left(\sum_{k}^{n}\left(\frac{\sum_{i}^{m} \sum_{j}^{n}\left(x_{i, j}^{\exp }-x_{i, j}^{c a l c}\right)^{2}}{6 n}\right)\right)^{1 / 2}$

donde $n$ es el número de líneas de reparto, el superíndice exp indica datos experimentales y el superíndice calc indica las concentraciones obtenidas usando el modelo de UNIQUAC. $m$ es la suma del número de componentes en cada una de las fases $(3+3)$ y $n$ es el total de líneas de reparto o equilibrios, (124) usadas para cada uno de los cuatro equilibrios ternarios estudiados en este trabajo. Es decir, se usaron 744 datos experimentales. Los cuales fueron usados para determinar los 38 parámetros binarios $\tau_{j i}$ de los equilibrios ternarios estudiados. Cada uno de los equilibrios fueron reportados a temperaturas de 25,35 y $45^{\circ} \mathrm{C}$. Estas temperaturas a las cuales fueron reportados los equilibrios son adecuadas para simular la separación de los productos de la fermentación estudiados en este trabajo (acetona, etanol, butanol y ácido acético). Adicionalmente, fueron incluidos equilibrios binarios de butanol y agua reportados a temperaturas entre 0 y $120^{\circ} \mathrm{C}$ reportados por Marongiu et al., 1984. 
El método de optimización de algoritmos genéticos ('ga' de Matlab ${ }^{\circledR}$ ) fue el método usado para la minimización de la función objetivo. Las condiciones por defecto de 'ga' fueron utilizadas en la minimización. Los parámetros de interacción binaria fueron hallados entre un rango de -5000 y 5000 . El método de algoritmo genético es propuesto es un método de búsqueda global (Rodríguez et al., 2016). No obstante, para obtener un set de parámetros adecuados, dado que 'ga' es un método metaheurístico, las minimizaciones fueron llevadas a cabo durante 10 veces. Con el fin de depurar aún más los resultados, los parámetros obtenidos en las minimizaciones usando 'ga' fueron posteriormente alimentados como las condiciones iniciales de un método de optimización no lineal local de MATLAB ${ }^{\circledR}$, 'Isqcurverfit'. Se seleccionaron los parámetros que alcanzan el valor de RMSD más bajo. Los parámetros obtenidos y su desempeño fueron comparados con anteriores reportes en la literatura de los equilibrios estudiados. Adicionalmente, fueron extraídos los parámetros binarios que tiene Aspen Plus $V 10.0^{\circledR}$, el software comercial más usado para la simulación de procesos químicos.

\section{RESULTADOS Y DISCUSIÓN}

Los resultados de las optimizaciones para el ajuste de los parámetros de las mezclas ternarias definidas se muestran en la Tabla 3. El resultado más bajo de RMSD obtenido usando algoritmos genéticos fue de 1.7 para la estimación de los equilibrios de butanol-agua- $2 \mathrm{E} 1 \mathrm{H}$, acetona-agua- $2 \mathrm{E} 1 \mathrm{H}$, etanol-agua- $2 \mathrm{E} 1 \mathrm{H}$, ácido acético-agua-2E1H. No obstante, cuando los resultados fueron depurados usando 'Isqcurverfit', el valor de la función objetivo (RMSD) durante la estimación fue reducido a $1.4 \%$. Resaltado la importancia de usar al menos dos métodos de optimización en el ajuste de parámetros.

Tabla 3: Parámetros ajustables de UNIQUAC encontrados en este trabajo

\begin{tabular}{|l|l|c|c|c|c|c|c|}
\hline Componente i & Componente $j$ & $a_{i j}$ & $a_{j i}$ & $b_{i j}$ & $b_{j i}$ & $c_{i j}$ & $c_{j i}$ \\
\hline Butanol & Agua & -26.70 & -32.37 & 747.1 & 2037.6 & 4.234 & 4.336 \\
\hline Butanol & 2E1H & -3.595 & 15.27 & 1505.3 & -4999.7 & 0 & 0 \\
\hline Acetona & Agua & -11.89 & 1.907 & 3401.3 & -608.5 & 0 & 0 \\
\hline Acetona & 2E1H & -15.17 & 1.470 & 4767.2 & -890.2 & 0 & 0 \\
\hline Etanol & Agua & 3.258 & -0.389 & -1098.4 & -1.893 & 0 & 0 \\
\hline Etanol & 2E1H & 5.533 & 0.028 & -1745.1 & -119.9 & 0 & 0 \\
\hline Ácido acético & Agua & 1.918 & -0.950 & -872.9 & 207.5 & 0 & 0 \\
\hline Ácido acético & 2E1H & 0.673 & -0.552 & -368.6 & 52.8 & 0 & 0 \\
\hline Agua & 2E1H & -2.51 & 1.920 & 780.7 & -1120.7 & 0 & 0 \\
\hline
\end{tabular}

Con el objetivo de analizar la capacidad para simular los equilibrios binarios líquido-líquido de butanol y agua a diferentes temperaturas, un análisis comparativo de los valores experimentales (Marongiu et al., 1984) y las simulaciones es mostrada en la Fig. 2. Tal como se observa en la Fig. 2, y de acuerdo con los resultados obtenidos de RMSD, los parámetros hallados en este trabajo fueron capaces de modelar tanto los equilibrios binarios de butanol-agua como los equilibrios ternarios objetos de estudio de este trabajo.

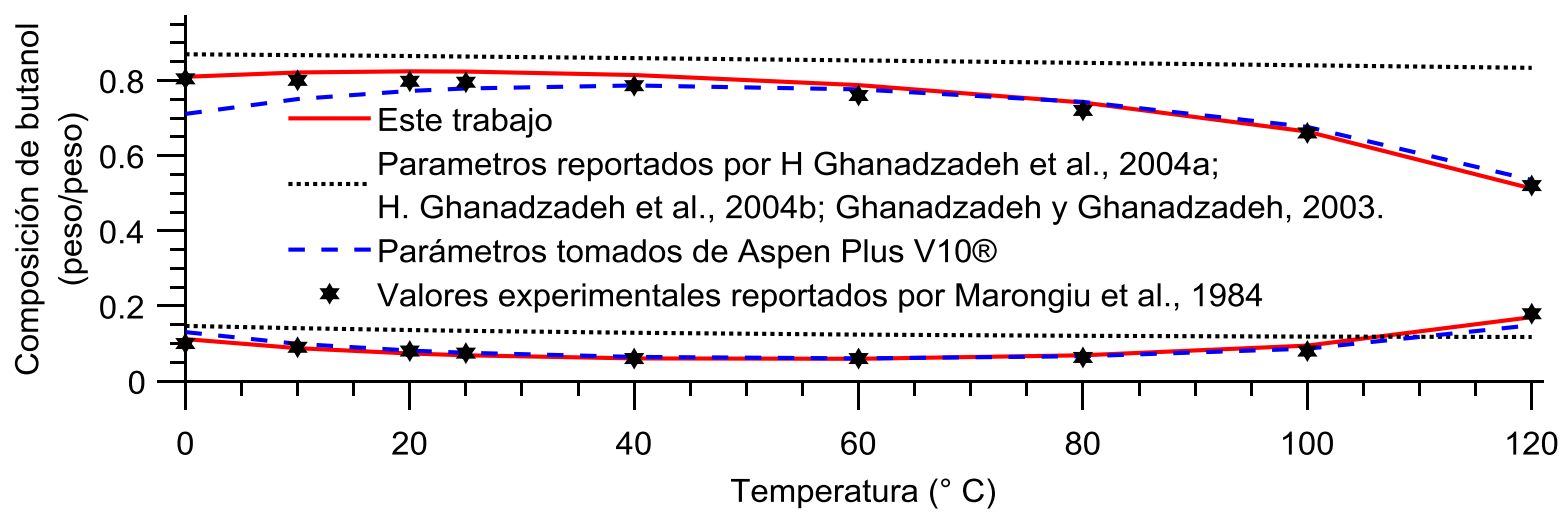

Fig. 2: Equilibrios binarios de butanol y agua a diferentes temperaturas.

Los parámetros obtenidos en este trabajo fueron comparados con dos sets de parámetros, los reportados en Aspen Plus ${\mathrm{V} 10^{\circledR}}^{\circledR}$ y los reportados por Ghanadzadeh et al., 2004a, 2004b; Ghanadzadeh y Ghanadzadeh, 2003 y Ghanadzadeh y Ghanadzadeh, 2004. Los diagramas de paridad usando los tres diferentes conjuntos de parámetros de interacción binaria son observados en la Fig. 3. Los resultados obtenidos en este trabajo se observan en la Fig. 3 (a). En el primer conjunto de parámetros que se usaron para comparar los resultados encontrados en este trabajo (Fig. 3 (b)), los equilibrios fueron determinados usando los parámetros de interacción binaria descritos en la Tabla 4. Ya que existen diferentes parámetros 
de interacción binaria reportados para 2E1H y agua, los parámetros usados fueron aquellos reportados por Ghanadzadeh y Ghanadzadeh, 2004, los cuales son reportados para butanol, ya que corresponden al valor más bajo de RMSD al evaluar los parámetros previamente reportados.
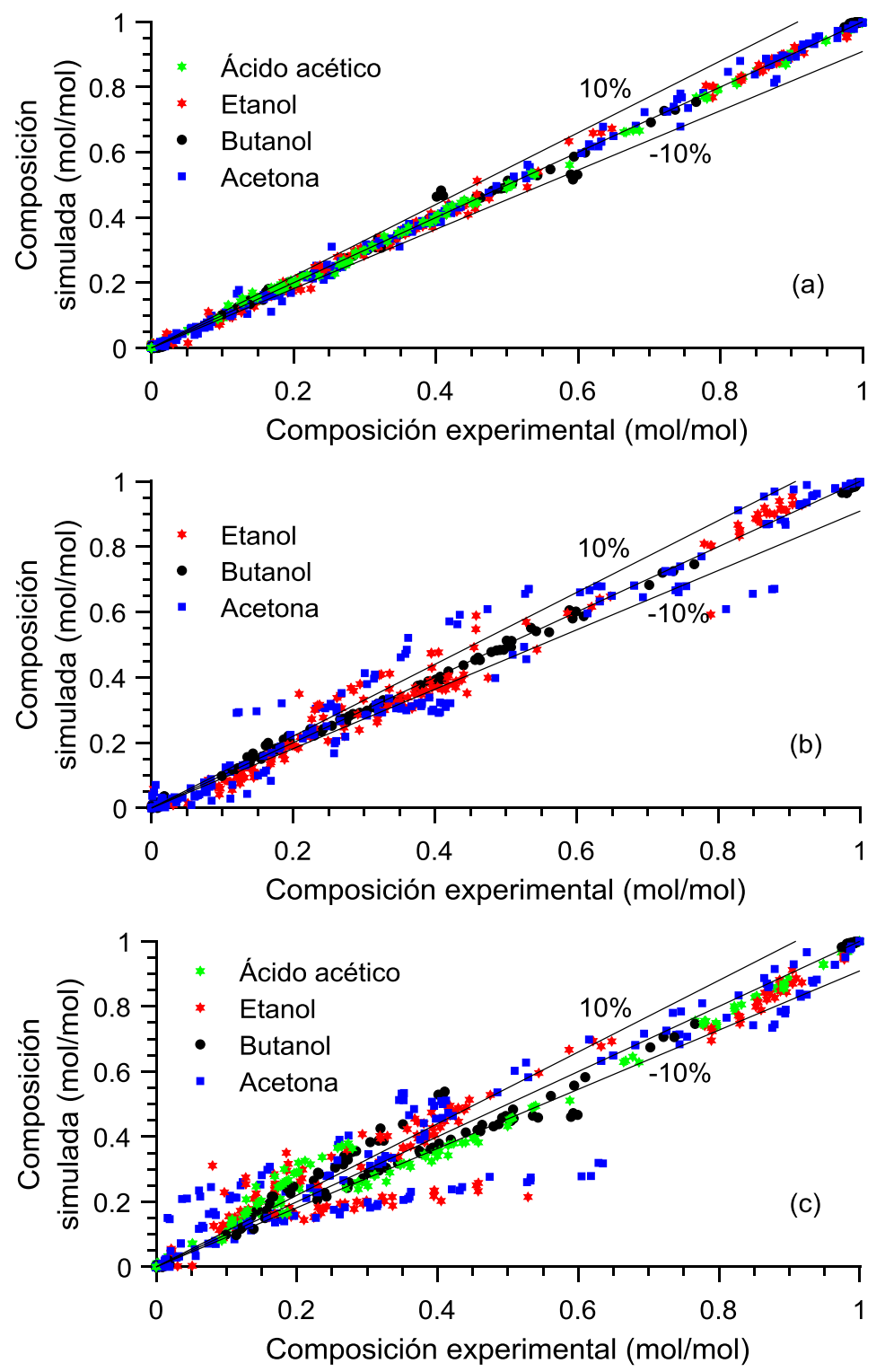

Fig. 3. Diagramas de paridad usando los parámetros binarios (a) los obtenidos en este trabajo, (b) los reportados por Ghanadzadeh et al., 2004a, 2004b; Ghanadzadeh y Ghanadzadeh, 2003, 2004 y (c) los extraídos de Aspen plus V10®

Tabla 4: Parámetros ajustables de UNIQUAC binaria reportados por Ghanadzadeh et al., 2004b; Ghanadzadeh y Ghanadzadeh, 2003; y Ghanadzadeh y Ghanadzadeh, 2004

\begin{tabular}{|l|l|l|l|l|l|l|l|l|}
\hline Componente i & Componente $j$ & $a_{i j}$ & $a_{j i}$ & $b_{i j}$ & $b_{j i}$ & $c_{i j}$ & $c_{j i}$ & Referencia \\
\hline Butanol & Agua & 0.50 & -1.04 & -340.9 & 226.1 & 0 & 0 & Ghanadzadeh y Ghanadzadeh, 2004 \\
\hline Butanol & 2E1H & 0.08 & -0.12 & 7.91 & -13.02 & 0 & 0 & Ghanadzadeh y Ghanadzadeh, 2004 \\
\hline Acetona & Agua & 5.24 & -4.93 & -1491.9 & 1302.8 & 0 & 0 & Ghanadzadeh et al., 2004b \\
\hline Acetona & 2E1H & 0.35 & 0.34 & -58.5 & -50.8 & 0 & 0 & Ghanadzadeh et al., 2004b \\
\hline Etanol & Agua & -0.99 & 0.81 & 328.1 & -446.0 & 0 & 0 & Ghanadzadeh y Ghanadzadeh, 2003 \\
\hline Etanol & 2E1H & 0.46 & -0.78 & -103.0 & 124.2 & 0 & 0 & Ghanadzadeh y Ghanadzadeh, 2003 \\
\hline Agua & 2E1H & 0.38 & -1.60 & -333.8 & 248.0 & 0 & 0 & Ghanadzadeh y Ghanadzadeh, 2004 \\
\hline
\end{tabular}

Con los valores reportados en la Tabla 4 fue alcanzado un RMSD (\%) de 2.8. Los diagramas de paridad de está simulación pueden ser observados en la Fig. 3 (b). Este RMSD (2.8\%) es 2 veces más alto que el encontrado en este trabajo. Es importante aclarar también que el equilibrio ternario involucrando ácido acético no fue estimado en este conjunto (Fig. 3 (b)), ya que no se encontraron parámetros binarios de UNIQUAC basados en estos datos experimentales. EI RMSD de este trabajo fue más bajo porque al 
considerar todos los equilibrios ternarios en el ajuste se estimaron unos parámetros de interacción de 2E1Hagua más realistas.

Con el objetivo de resaltar la necesidad de hacer un ajuste de datos para simular los cuatro equilibrios ternarios estudiados, se realizó una comparación con las simulaciones de los equilibrios usando un segundo conjunto de parámetros (Fig. 3 (c)), los cuales están reportados en Aspen Plus V10 ${ }^{\circledR}$, ver Tabla 5. Es importante recalcar que las simulaciones usando los parámetros de Aspen Plus $V 10^{\circledR}$ son predicciones, ya que los parámetros de interacción binaria son obtenidos en su mayoría a partir de equilibrios binarios y no ternarios, tal como fue realizado en este trabajo. Por otro lado, los datos experimentales usados en este trabajo no están reportados en la base de datos de Aspen Plus V10 ${ }^{\circledR}$. Adicionalmente, en Aspen Plus V10 ${ }^{\circledR}$, no todos los parámetros de interacción binaria de UNIQUAC son basados en datos experimentales y estos son predichos a partir de un modelo de actividad de grupos funcionales, UNIFAC.

Con relación al equilibrio líquido-líquido de butanol agua a diferentes temperaturas, se encontró que los parámetros reportados en Aspen Plus $\mathrm{V}_{10} 0^{\circledR}$ son adecuados, lo que era de esperarse ya que en Aspen Plus V $10^{\circledR}$ los parámetros son obtenidos principalmente a partir de equilibrios binarios. Las simulaciones que usaron este conjunto de parámetros alcanzaron las más altas desviaciones con respecto a los valores experimentales, logrando un RMSD 4.7 veces más grande que el encontrado en este trabajo. Estos resultados muestran que el modelo de UNIQUAC tiene cierta capacidad predictiva, i.e. el RMSD fue de $6.6 \%$. No obstante, la necesidad de obtener el un nuevo set de parámetros de interacción binaria de este trabajo usando equilibrios ternarios es evidenciado por la reducción que se logró en el RMSD.

Tabla 5: Parámetros de interacción binaria de UNIQUAC reportados en Aspen Plus V10 ${ }^{\circledR}$

\begin{tabular}{|l|l|l|l|l|l|l|l|}
\hline Componente i & Componente $j$ & $a_{i j}$ & $a_{j i}$ & $b_{i j}$ & $b_{j i}$ & $c_{i j}$ & $c_{j i}$ \\
\hline Butanol & Agua & -70.97 & 6.63 & 3132.8 & -16.5 & 10.63 & -1.323 \\
\hline Butanol & 2E1H & 1.425 & -0.962 & -880.0 & 575.5 & 0 & 0 \\
\hline Acetona & Agua & 3.051 & -2.607 & -1380.1 & 924.2 & 0 & 0 \\
\hline Acetona & E1H & 0 & 0 & 54.4 & -163.0 & 0 & 0 \\
\hline Etanol & Agua & 2.412 & -2.749 & -898.6 & 872.3 & 0 & 0 \\
\hline Etanol & 2E1H & 0 & 0 & 37.8 & -117.5 & 0 & 0 \\
\hline Ácido acético & Agua & 0 & 0 & 219.7 & -73.4 & 0 & 0 \\
\hline Ácido acético & 2E1H & 0 & 0 & 199.2 & -112.4 & 0 & 0 \\
\hline Agua & 2E1H & -0.7007 & 0.7694 & -42.8 & -518.3 & 0 & 0 \\
\hline
\end{tabular}

\section{CONCLUSIONES}

Usando UNIQUAC y los parámetros ajustados en este trabajo se alcanzó un RMSD de 1.4\% para simular los equilibrios ternarios de butanol-2E1H-agua, etanol-2E1H-agua, acetona- $2 \mathrm{E} 1 \mathrm{H}$-agua, ácido acético- $2 \mathrm{E} 1 \mathrm{H}$ agua. EI RMSD alcanzado en este trabajo fue 4.7 veces menor que los obtenidos usando los parámetros binarios reportados en Aspen Plus $\mathrm{V} 10^{\circledR}$ y 2 veces más bajo que los parámetros ajustables de UNIQUAC reportados por H Ghanadzadeh et al., 2004; H. Ghanadzadeh et al., 2004; Ghanadzadeh y Ghanadzadeh, 2003. A partir de los resultados de este trabajo, se recomienda usar la mayor cantidad disponible de datos experimentales de equilibrios líquido-líquido para poder alcanzar simulaciones con el más bajo error posible y no se recomienda el uso de los parámetros disponibles de Aspen Plus V10 ${ }^{\circledR}$ para la extracción de butanol mediante $2 \mathrm{E} 1 \mathrm{H}$. En este caso de estudio, fue encontrado que usar dos métodos secuenciales de optimización logra reducir el RMSD de 1.7 a 1.4\%, una reducción de 18\% en el RMSD.

\section{AGRADECIMIENTOS}

Los autores agradecen a Colciencias pues este trabajo fue financiado gracias a la convocatoria de estancia postdoctoral 811 de COLCIENCIAS, Colombia 2018. Adicionalmente, agradecemos a Colciencias por soportar la publicación de este articulo a través del proyecto "Modelado y simulación del Metabolismo Urbano de Bogotá D.C. Código 111974558276 ".

\section{REFERENCIAS}

Abrams, D.S. y Prausnitz, J.M., Statistical Thermodynamics of Liquid Mixtures: A New Expression for the Excess Gibbs Energy of Partly or Completely Miscible Systems, https://doi.org/10.1002/aic.690210115, AIChE J., 21, 116-128 (1975)

Bedade, D.K., Dev, M.J. y Singhal, R.S., Bioreactor Studies on Acrylamidase Produced from Cupriavidus oxalaticus ICTDB921: Production, Kinetic Modeling, and Purification, Biochem. Eng. J., 149, 107245 (2019)

Boock, J.T., Freedman, A.J.E. y otros ocho autores, Engineered Microbial Biofuel Production and Recovery under Supercritical Carbon Dioxide, https://doi.org/10.1038/s41467-019-08486-6, Nat. Commun., 10, 587 (2019) 
Dalle-Ave, G. y Adams, T.A., Techno-Economic Comparison of Acetone-Butanol-Ethanol Fermentation using Various Extractants, https://doi.org/10.1016/j.enconman.2017.11.020, Energy Convers. Manag., 156, 288-300 (2018)

Davison, B.H. y Thompson, J.E., Continuous Direct Solvent Extraction of Butanol in a Fermenting Fluidized-Bed Bioreactor with Immobilized Clostridium acetobutylicum, Appl. Biochem. Biotechnol., 39-40, 415-426 (1993)

Forero, L.A. y Velásquez, J.A., Representación Simultánea del Equilibrio Líquido-Vapor, el Volumen Molar y la Entalpía de Exceso de Mezclas Complejas Mediante una Ecuación de Estado tipo Peng-Robinson, https://doi.org/10.4067/s071807642019000600021, Inf. Tecnol., 30, 21-34 (2019)

Ghanadzadeh, H. y Ghanadzadeh, A., (Liquid+liquid) Equilibria in (water+ethanol+2-ethyl-1-hexanol) at $T=(298.2,303.2$, 308.2, and 313.2), https://doi.org/10.1016/S0021-9614(03)00127-7, J. Chem. Thermodyn.. 35, 1393-1401 (2003)

Ghanadzadeh, H., Ghanadzadeh, A. y Sariri, R., (Liquid+liquid) Equilibria for (water+acetic acid+2-ethyl-1-hexanol): experimental data and prediction, J. Chem. Thermodyn., 36, 1001-1006 (2004a)

Ghanadzadeh, H. y Ghanadzadeh, A., Liquid-Liquid Equilibria of Water + 1-Butanol + 2-Ethyl-1-hexanol System, https://doi.org/10.1021/je030202l, J. Chem. Eng. Data, 49, 783-786 (2004)

Ghanadzadeh, H., Ghanadzadeh A. y Alitavoli, M., LLE of Ternary Mixtures of water/acetone/2-ethyl-1-hexanol at Different Temperatures, https://doi.org/10.1016/j.fluid.2004.02.006, Fluid Phase Equilib., 219, 165-169 (2004b)

González-Peñas, H., Lu-Chau, T.A. y otros dos autores, Solvent Screening Methodology for in situ ABE Extractive Fermentation, https://doi.org/10.1007/s00253-014-5634-6, Appl. Microbiol. Biotechnol., 98, 5915-5924 (2014)

Grisales Díaz, V. H., von Stosch, M., y Willis, M. J., Butanol Production via Vacuum Fermentation: An Economic Evaluation of Operating Strategies, https://doi.org/10.1016/j.ces.2018.10.016, Chemical Engineering Science, 195, 707-719 (2019)

Grisales-Díaz, V.H. y Olivar-Tost, G., Economic Optimization of in situ Extraction of Inhibitors in Acetone-Ethanol-Butanol (ABE) Fermentation from Lignocellulose, https://doi.org/10.1016/j.procbio.2018.04.014, Process Biochem., 70, 1-8 (2018)

Grisales-Díaz, V.H. y Olivar-Tost, G., Techno-Economic Analysis of Extraction-Based Separation Systems for Acetone, Butanol, and Ethanol Recovery and Purification, https://doi.org/10.1186/s40643-017-0142-z, Bioresour. Bioprocess., $4,12$. (2017)

Groot, W.J., Soedjak, H.S. y otros cuatro autores, Butanol Recovery from Fermentations by Liquid-Liquid Extraction and Membrane Solvent Extraction, Bioprocess Eng., 5, 203-216 (1990)

Groot, W.J., van der Lans, R.G.J.M. y Luyben, K.C.A.M., Technologies for Butanol Recovery Integrated with Fermentations, https://doi.org/10.1016/0032-9592(92)80012-R, Process Biochem., 27, 61-75 (1992)

Karimi-Alavijeh, M. y Karimi, K., Biobutanol Production from Corn Stover in the US, https://doi.org/10.1016/j.indcrop.2018.12.054, Ind. Crops Prod., 129, 641-653 (2019)

Leo, A., Hansch, C. y Elkins, D., Partition Coefficients and their Uses, Chem. Rev., 71, 525-616 (1971)

Lu, K. y Li, S., An Integrated in situ Extraction-Gas Stripping process for Acetone-Butanol-Ethanol (ABE) Fermentation, https://doi.org/10.1016/j.jtice.2014.06.023, J. Taiwan Inst. Chem. Eng. 45, 2106-2110 (2014)

Marongiu, B., Ferino, I. y otros tres autores, Thermodynamic Properties of Aqueous Non-Electrolyte Mixtures. Alkanols ++ Water Systems, https://doi.org/10.1016/0167-7322(84)80027-6, J. Mol. Liq., 28, 229-247 (1984)

Matsumura, M., Kataokay, H. y otros dos autores, Energy Saving Effect of Pervaporation using Oleyl Alcohol Liquid Membrane in Butanol Purification, https://doi.org/10.1007/BF00369334, Bioprocess Eng. 3, 93-100 (1988)

Naidoo, M., Tai, S.L. y Harrison, S.T.L., Energy Requirements for the in-situ Recovery of Biobutanol via Gas Stripping, https://doi.org/10.1016/j.bej.2018.07.022, Biochem. Eng. J. 139, 74-84 (2018)

Outram, V., Lalander, C.-A. y otros tres autores, A Comparison of the Energy use of in situ Product Recovery Techniques for the Acetone Butanol Ethanol Fermentation, Bioresour. Technol., 220, 590-600 (2016)

Qureshi, N., Hughes, S. y otros dos autores, Energy-Efficient Recovery of Butanol from Model Solutions and Fermentation Broth by Adsorption, https://doi.org/10.1007/s00449-005-0402-8, Bioprocess Biosyst. Eng., 27, 215-222 (2005)

Rezaei, H., Abdolrahimi, S. y otros dos autores, A Nonextensive Electrolyte UNIQUAC Model for Prediction of Mean Activity Coefficients of Binary Electrolyte Solutions, Phys. Chem. Res., 6, 773-784. (2018)

Rodriguez, A.B., Saavedra, F. y Ramírez, L.J., Estrategia Metaheurística para Redes Ópticas sin Conversión de Longitud de Onda con Tráfico Dinámico (WDM), Inf. Tecnol., 27, 221-230, (2016)

Singh, V., Singh, H. y Das, D., Optimization of the Medium Composition for the Improvement of Hydrogen and Butanol Production using Clostridium Saccharoperbutylacetonicum DSM 14923, Int. J. Hydrogen Energy, 44, 26905-26919 (2019)

Sørensen, J.M., Magnussen, T. y otros dos autores, Liquid-liquid equilibrium data: Their Retrieval, Correlation and Prediction Part II: Correlation, https://doi.org/10.1016/0378-3812(79)80027-8, Fluid Phase Equilib., 3, 47-82. (1979)

Thomsen, K., Ciliuta, M. y otros tres autores, Extended UNIQUAC Model for Correlation and Prediction of Vapor-LiquidLiquid-Solid Equilibria in Aqueous Salt Systems Containing Non-Electrolytes. Part B. Alcohol (ethanol, propanols, butanols)water-salt systems, https://doi.org/10.1016/j.ces.2004.05.024, Chem. Eng. Sci., 59, 3631-3647 (2004). 